\title{
The Cupola
}

\section{Scholarship at Gettysburg College}

$7-1987$

\section{Membership in the Young Cluster Trumpler 37}

Laurence A. Marschall

Gettysburg College

William F. Van Altena

Yale University

Follow this and additional works at: https:// cupola.gettysburg.edu/physfac

Part of the Stars, Interstellar Medium and the Galaxy Commons

Share feedback about the accessibility of this item.

Marschall, Laurence A., and William F. Van Altena. Membership in the Young Cluster Trumpler 37. The Astronomical Journal (July 1987) $94(1): 71-83$.

This is the publisher's version of the work. This publication appears in Gettysburg College's institutional repository by permission of the copyright owner for personal use, not for redistribution. Cupola permanent link: https://cupola.gettysburg.edu/physfac/105

This open access article is brought to you by The Cupola: Scholarship at Gettysburg College. It has been accepted for inclusion by an authorized administrator of The Cupola. For more information, please contact cupola@gettysburg.edu. 


\title{
Membership in the Young Cluster Trumpler 37
}

\begin{abstract}
Astrometric positions for 1387 stars and proper motions for a subset of 1135 stars brighter than $V=15$ in a 1.5 degree surrounding the young open cluster Trumpler 37 are presented. Membership probabilities are presented for those stars with measured proper motions, and 486 stars with probabilities of $>80 \%$ are identified.
\end{abstract}

Keywords

Trumpler 37, open cluster stars

\section{Disciplines}

Astrophysics and Astronomy | Stars, Interstellar Medium and the Galaxy 


\title{
MEMBERSHIP IN THE YOUNG CLUSTER TRUMPLER 37
}

\author{
LAGRENCE A. MARSCHAI.I. \\ Department of Physics. Gettysburg College. Getusburg. Pennsylvania 17325 \\ WILLIAM F, VAN ALTIENA \\ Yale Unversity Ohsersatory. New Hawen, Connecticut 06511 \\ Receired 5 warch lgs7 revised 3 april lys?
}

\begin{abstract}
Astrometric positions for 1387 stars and proper motions for a subset of 1135 stars brighter than $V=15$ in a $1.5^{\circ}$ field surrounding the young open cluster Trumpler 37 are presented. Membership probabilities are presented for those stars with measured proper motions. and 486 stars with probabilities of $>80 \%$ are identified.
\end{abstract}

\section{INTRODUCTION}

The open cluster Trumpler 37 (Co $2137-572, l=99.3^{\circ}$. $\left.b=3.73^{\circ}\right)$, identified as the nucleus of the Cepheus OB 2 association (Simonson 1968), is one of the very youngest clusters $(\log \tau<6.5)$, and its distance of less than $1 \mathrm{kpc}$ makes it also one of the nearest of its age group. Yet it has received little attention in studies of stellar evolution compared to other clusters of comparable ages such as IC 1805. NGC 2244, NGC 2264, and NGC 6530 (Lynga 1985). Of these, it most nearly resembles NGC 2244, which is embedded in the central "hole" of the Rosette Nebula. Tr 37 similarly lies in a region of reduced emission surrounded by the $H$ II region IC 1396. Barnard's Milky Way atlas (1927) describes it thus: "The very center of these nebulous masses is gritty with small stars which are entirely free of nebulosity. All around this stars for some distance are veiled in nebulosity:" On the Palomar Sky Survey red photograph, the nebula appears exceedingly Rosette-like, and though it is larger in angular size, this is primarily due to the greater distance of the Rosette. Actually, both the nebula and the cluster are quite comparable to the Rosette in physical extent. A number of recent radio studies of IC 1396 have noted this similarity (Wendker and Baars 1980; Heske and Wendker 1986).

As listed in Lynga's cluster catalog, both NGC 2244 and Tr 37 show similar amounts of visual absorption, about 1.5 mag. The relative neglect of $\operatorname{Tr} 37$ by astronomers to date may be due in part to its general lack of concentration, accentuated by its larger angular extent on the sky. Lynga classifies Tr 37 as a Trumpler class IV object, less distinct from the background than NGC 2244, which he assigns a Trumpler class II. In his original survey of clusters, Trumpler (1930) assigned them both to class IV, and noted that $\operatorname{Tr} 37$ seems to be a more populous grouping of stars.

The central star of the cluster, HD 206267, was included in a recent study of "Trapezium-like" systems by Abt (1986). It is one of the youngest examples of these multiplestar systems, which Abt likens to the Trapezium ( $\theta^{1}$ Orionis) in having components of nearly identical separation. Though not quite as complex and active a region as Orion, the interaction between stars and gas is still quite evident in the vicinity of the cluster (Wendker and Baars 1980). That, together with the relative youth and proximity of Trumpler 37 , promises interesting results for studies of the early evolution of stars. However, fundamental observational data is spotty.

Alksinis (1958) has published an extensive set of magnitudes $\left(m_{\mathrm{ng}}\right)$ and approximate spectral types (no luminosity classes are given) for stars in the Cepheus region, including about a hundred members of the cluster, but these have not been tied to photoelectric $U B V$ sequences or modern $\mathrm{MK}$ spectral types. Only a few of the brighter stars in the cluster were included in a spectroscopic and photometric study of the Cepheus association by Simonson (1968). The only focused scrutiny of the cluster remains the paper of Garrison and Kormendy (1976), who published MK classifications for 51 stars and photoelectric $U B V$ photometry for 37 stars brighter than $V=10.1$ in a region about $3^{\circ}(48 \mathrm{pc}$ ) in diameter surrounding $\operatorname{Tr} 37$. They derive a distance modulus of 9.9 for the cluster. and their earliest main-sequence spectral type of $\mathrm{O} 6$ indicates that the cluster is comparable in age (2$\left.4 \times 10^{\circ} \mathrm{yr}\right)$ to the very youngest open clusters, such as $\mathrm{NGC}$ 2244 (Marschall et al. 1982) and NGC 6530 (van Altena and Jones 1972). The Garrison and Kormendy photometry has been supplemented by photoelectric $U B V R I$ magnitudes of 26 fainter cluster stars $10.5<V<15.75$ by Cardon de Lichtbuer (1982). Most recently, Kun (1986) has identified $155 \mathrm{H} \alpha$ emission stars fainter than $V=13$ in a 19.5 square degree field centered on $\operatorname{Tr} 37$ (approximately the same coverage as Garrison and Kormendy), and has presented photographic $U B V R$ magnitudes for them.

No comprehensive membership studies of the cluster have been undertaken previously. This study presents measurements of astrometric positions of 1387 stars along with proper motions for 1135 stars brighter than $V=15$ within approximately $0.75^{\circ}$ (12.5 pc) of the cluster center. The astrometric positions and membership probabilities for $\mathrm{Tr}$ 37 should establish a foundation for further photometric and spectroscopic studies of its member stars, adding to the roster of nearby young clusters suitable for studies of stellar evolution.

\section{PLATE MEASUREMENT AND REDLCTION}

The plate material for this study consisted of 17 Yerkes plates covering seven overlapping fields centered on the star HD 206267 (R.A. (1950) $=21^{\mathrm{h}} 37^{\mathrm{m}} 24^{\mathrm{s}}$, Dec. (1950) $\left.=57^{\circ} 15^{\prime} 45^{\prime \prime}\right)$. Table I lists pertinent data for the plates, and Fig. 1 [Plate 5] shows the coverage of the overlapping fields. We refer to these as the center field, east field, northeast field. etc. All star images on the plates, a region roughly $1.5^{\circ}$ in diameter, were measured using the PDS microdensitometer at Yale University Observatory. Positions and magnitudes for each image were reduced using a two-dimensional Gaussian fitting program written by Jin-Fuw Lee for the PDP $11 / 60$ computer at Yale. 
TABI.E I. Yerkes Observatory plate material for Tr 37

\begin{tabular}{|c|c|c|c|}
\hline Field & Plate ID & Date & Exposure \\
\hline Center & 1567 & July 22, 1937 & $36 \mathrm{~min}$. \\
\hline Center & F373 & July 24,1937 & $22 \mathrm{~min}$. \\
\hline Center & $\pi 24169$ & Aug. $2,19 \% 3$ & $30 \mathrm{~min}$. \\
\hline Center & $\pi 24185$ & Aug. 4, 1973 & 25 שin. \\
\hline East & F 374 & July 24,1937 & $35 \mathrm{~min}$. \\
\hline East & $\pi 24186$ & Aug. 4,1973 & 25 min. \\
\hline East & $\pi 24275$ & Oct. 19,1973 & 30 min. \\
\hline Northeast & $\$ 370$ & July 22, 1937 & $30 \mathrm{~min}$. \\
\hline Northeast & $\pi 24189$ & Aug. 5,1973 & $25 \mathrm{~min}$. \\
\hline Northwest & F369 & July $22,193 i$ & $18 \mathrm{~min}$. \\
\hline Northwest & $\pi 24274$ & Oct. 19,1973 & $30 \mathrm{~min}$. \\
\hline West & F368 & July 22, 1937 & $25 \mathrm{~min}$. \\
\hline Wiest & T. 24184 & Aug. 4, 1973 & $25 \mathrm{~min}$ \\
\hline Southwest & F381 & Juiy 26,1937 & $20 \mathrm{~min}$ \\
\hline Southwest & $r: 24187$ & Aug. 5,1973 & 25 min. \\
\hline Southeast & F 380 & July 26, 1937 & $25 \mathrm{~min}$. \\
\hline Southeast & $\pi 24183$ & Aug. 4, 1973 & 25 min. \\
\hline
\end{tabular}

Proper motions were computed by an iterative leastsquares-fitting procedure as described in our study of NGC 2244 (Marschall et al. 1982). Proper motions for each separate field were computed independently. Thus the solutions for the center field involved four plates, two first epoch (1937) and two second epoch (1973); the east field involved three plates, one first epoch and two second epoch, and all the other fields used a pair of plates. In each case, linear and quadratic terms in the coordinates and magnitude were used, but since color measurements were unavailable for most of the stars, no color terms were included; nor were they found necessary in our previous study, which used both Yerkes and Allegheny plates.

The proper motions derived for each separate field were transformed to the proper motions of stars on the central plate with a linear least-squares fit involving the overlapping stars in each field; only rotation, translation, and scale change were included in the fit. These consolidated values of the proper motions were then used to derive membership probabilities. The consolidated proper-motion vector-point diagram is shown in Fig. 2. The limited number of plates used in each solution compared to our study of NGC 2244 , which involved 18 plates, and the smaller epoch difference, $36 \mathrm{yr}$ as opposed to $57 \mathrm{yr}$, produces a scatter in proper motions that is greater than in our previous work. There is nevertheless a clear concentration of points near zero relative motion which represents the cluster against a more widely dispersed background of field-star motions. The separate distributions are more clearly recognizable on the marginal histograms of the proper motions shown in Fig. 3.

The field and cluster distributions were derived using a program supplied by Dr. E. Green. The program fits twodimensional Gaussians to both the field and cluster propermotion distributions, with due account taken for the propermotion errors of each individual star. Where more than two plates were used in the solutions, the errors used as input to the program were derived from the residuals in the plate solution. In the two-plate solutions, no such formal error was produced (the residuals in these cases are attributed

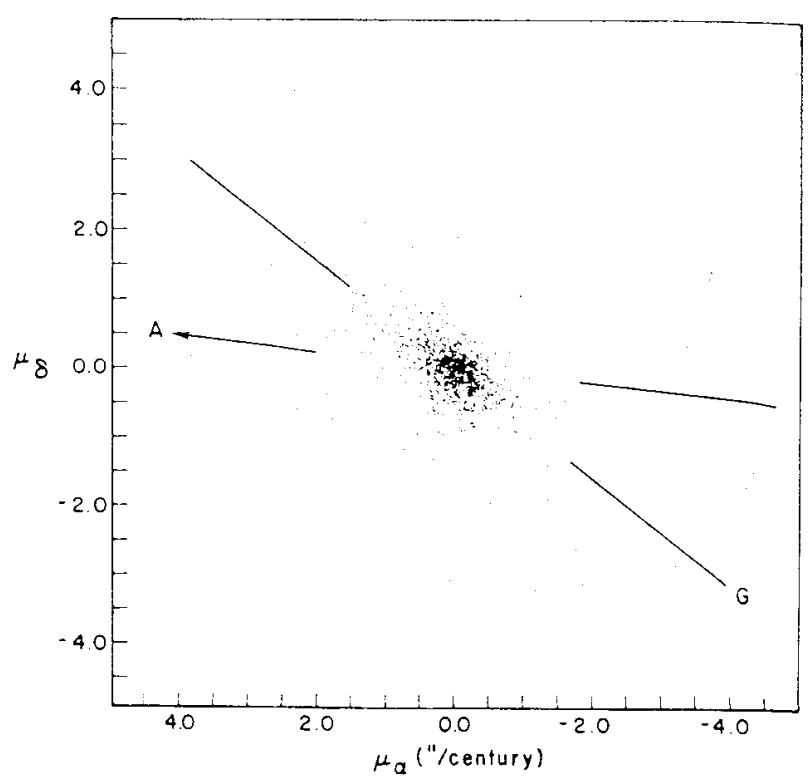

FIci. 2. Observed proper-motion vector-point diagram for Trumpler 37. The direction toward the solar apex and a line parallel to the galactic plane are indicated. The units are in arcseconds per century.
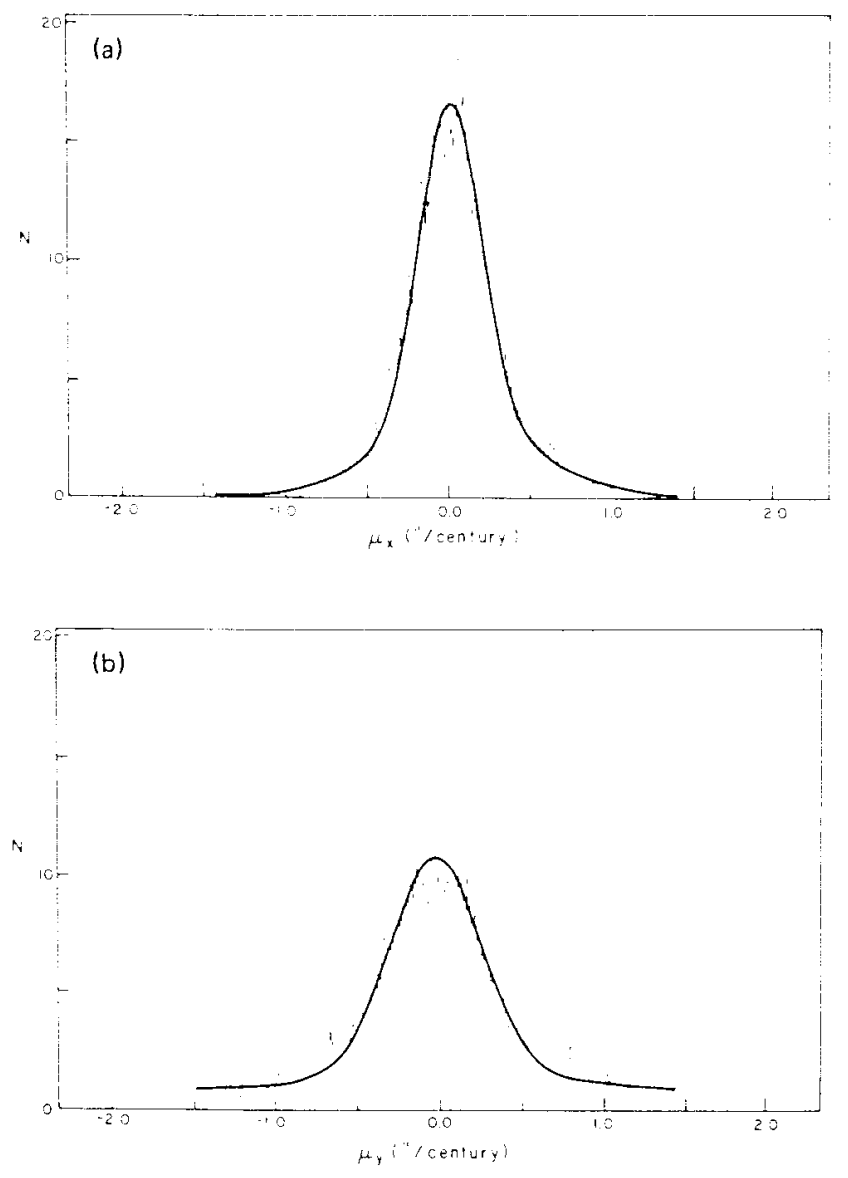

Fic. 3. Marginal distributions of the vector-point diagram rotated $48^{\circ}$ to align $X$ and $Y$ with the minor and major axes of the field distribution. The observed distributions are drawn with a thin line, while the conputed fits are draw $n$ with a heavy solid line. (a) Histogram for $X$ motions. (b) Histogram for $Y$ motions. 
entirely to proper motion, so the error was determined by assuming that the error for a single star was the same as the average for stars of similar magnitude in the multiple-plate solutions scaled to one plate pair.

The fitting program allows one to hold field parameters fixed while searching for a best fit to the cluster parameters. It was found, in accord with Cudworth's analysis of M22 (Cudworth 1986), that a Gaussian was not the best fit to the field-star distribution, and the final fits we present represent a compromise between underemphasizing the peak or overemphasizing the wings of the fitted field distribution. We favored the latter extreme, noting, as Cudworth does, that the derived probabilities are more sensitive to the values of the field-star distribution in the vicinity of the cluster proper-motion peak.

Parameters for cluster and field-star fits are listed in Table I1. The proper-motion centers and standard deviations are listed in arcseconds per century. The standard deviations listed for the cluster are a measure of the internal precision of the derived proper motions. not of the internal velocity dispersion of the cluster, which should be smaller by almost an order of magnitude as we discuss later in this paper. The precision is about 4 times less than that of our study of $\mathrm{NGC}$ 2244. That study utilized 18 plates in the solution, however. so the results for $\operatorname{Tr} 37$ are consistent with the measurement errors for a single star in the NGC 2244 study.

\section{POSITIONS AND MEMBERSHIP PROBABILITIES}

The final results of the plate measurement and propermotion analysis are listed in Table III. Numbers in column 1 refer to the identification charts in Figs. $4(\mathrm{a})-(\mathrm{g})$ IPlates 6-12]. Coordinates in columns 2 and 3 were computed with transformations found by fitting the measurements of a dozen stars to positions given in the AGK3 catalog. The listed coordinates are epoch 1950 and refer to the equator and equinox of 1950 . They should be reliable to 0 ". 1. except for stars that have no derived proper motion as noted below. Proper motions in columns 4 and 5 are given in seconds of are per century. Proper motions are not given for stars where a good image center could be measured on only one plate. This was the case primarily for faint stars that did not appear on the first-epoch exposures. $V$ magnitudes in column 6 were derived by fitting photometric magnitudes from Garrison and Kormendy (1976) and Cardon de Lichtbuer (1982) to instrumental magnitudes derived from the plate scans. These magnitudes should be used with caution: they are only provided to aid in identifying stars on our charts. While the fit of instrumental to observed magnitudes is good to $0.1 \mathrm{mag}$ for the fainter stars $(V>10)$, the PDS photometry reduction we used did not account for saturation in the densest images. systematically affecting the precision of fit for the brighter stars. These may be in error by 0.3 mag. We have made additional photoelectric measurements of about one

TaBie 11 Membership probability parameters for Tr 37

\begin{tabular}{|c|c|c|}
\hline Paratater & Cluster & Field \\
\hline $\begin{array}{l}\text { Sumber } \\
u_{x} \text { center } \\
y_{y} \text { center } \\
y_{x} \text { dispersiun } \\
y_{y} \text { dispersion }\end{array}$ & $\begin{aligned} & 655 \\
+ & 0.101 \text { cent. } \\
- & 0.02 \\
\pm & 0.19 \\
\pm & 0.28\end{aligned}$ & $\begin{aligned} & 543 \\
+ & 0.00 \text {.cent. } \\
+ & 0.09 \\
\pm & 0.50 \\
\pm & 1.50\end{aligned}$ \\
\hline
\end{tabular}

hundred stars in the cluster, and will publish these data separately when we have accumulated a more complete set of measurements.

Probabilities of cluster membership, computed from the fitted cluster parameters, are listed in column 7 , and a quality estimate is listed in column 8. This quality estimate was made as follows. For all stars in the center and east fields (i.e., those with multiple-plate solutions), a quality class of 1 was assigned to those whose residuals from the plate solutions were less than 0.08 arcsec/century ( $\sigma$ for fitting of the center plate series), and a quality class of 2 to those with higher residuals. In all other fields, a quality class of 1 was assigned to all stars brighter than $V=14.5$, since this is where the scatter in the residuals for the multiple-plate solutions became significantly greater.

\section{DISCLSSION}

A histogram of the membership probabilities is shown in Fig. 5, illustrating a clear differentiation between field and cluster stars. Since the internal errors in this study are greater than in our previous ones, greater caution must be used in applying these numbers, however, and we would urge that only stars with $P>80 \%$, that is, 486 stars in all, be considered potential cluster members. That this criterion is consistent with the photometric results is borne out by its inclusion of the brighter $O$ and $B$ stars in the area, which are surely cluster members, and its rejection of the brighter stars identified with late spectral types in Alksinis' 1958 study. Kun (1986) has noted that bright early-type stars in this region seem dispersed over an area far larger than that of a typical cluster, and suggests that they may not all be part of the same group, or that the system may not be gravitationally bound. Thus the latter criterion, the rejection of brighter late-type stars, is likely to be more selective than the inclusion of bright early-type stars. as is indicated by our derived probabilities

More specifically, 149 stars in our study have been identified with those in Alksinis' study. A cross reference to the identification numbers in both studies is given in Table IV; the stars studied by Alksinis are designated Alks, the numbers in the current study MVA. Seven of these stars yielded only positions in the current study, the remaining 142 both positions and proper motions. Of 35 stars in the field with spectral classes earlier than AO according to Alksinis, 25 , or $71 \%$, are probable kinematic cluster members. A notable exception is HD 206267 itself (Alks 466, MVA 460 ), which has a computed membership probability of 67. This star, having the earliest spectral type in the region, is surely a cluster member, as we note in a footnote to Table III. The image of this multiple system suffers from blending of the components (with star MVA 461) on the first-epoch plates, producing a spurious determination of the image center. This is the only case in this study for which image blending is severe.

Of 49 stars with spectral types later than FO in Alksinis study, $42(86 \%)$ turn out to be kinematic nonmembers. Some of the remaining seven stars (MVA $550.555,800,883,1050,1166$, and 1539 ) may be chance coincidences or spectroscopic misidentifications, but they also deserve further scrutiny as possible evolved or pre-main-sequence members of the cluster. Three radial velocities of MVA 550 obtained by one of us (LAM) with the echelle spectrometer of the Fred L. Whipple Observatory and the Multiple Mirror Telescope indicate that it is a single-line 
TABLF III. Observational data for stars in $\operatorname{Tr} 37$

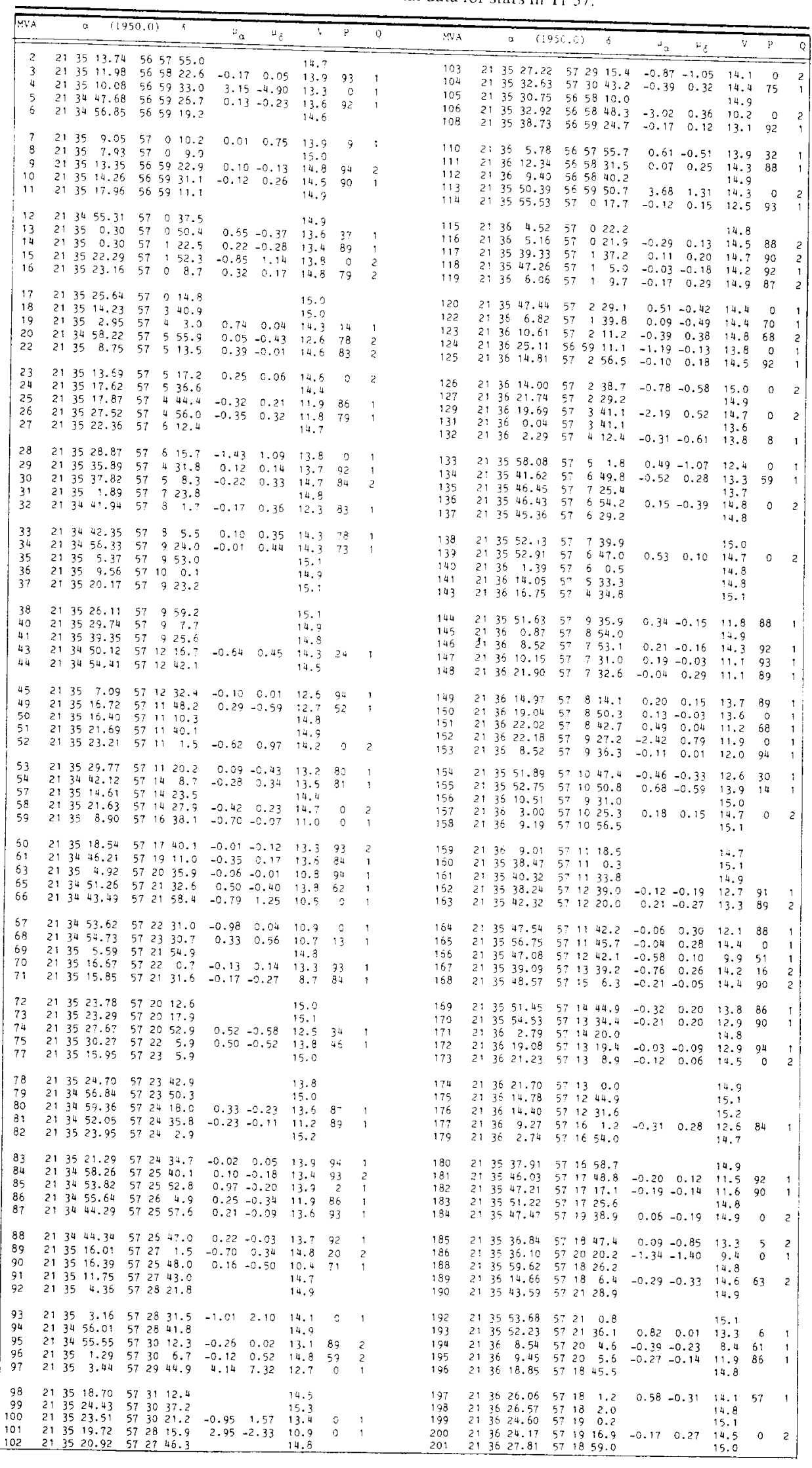


TABL.F III. (continued)

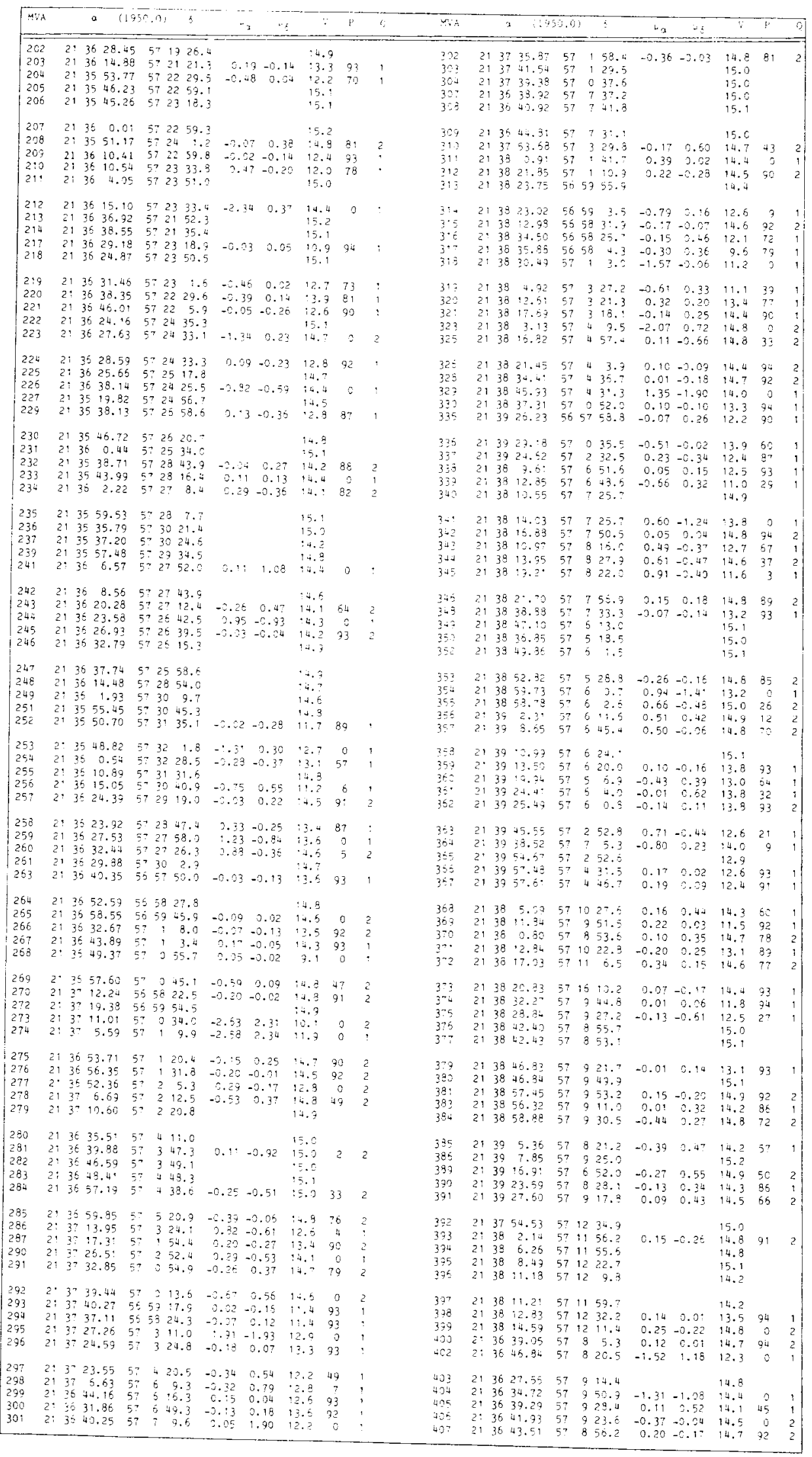


TABLE III. (continued)

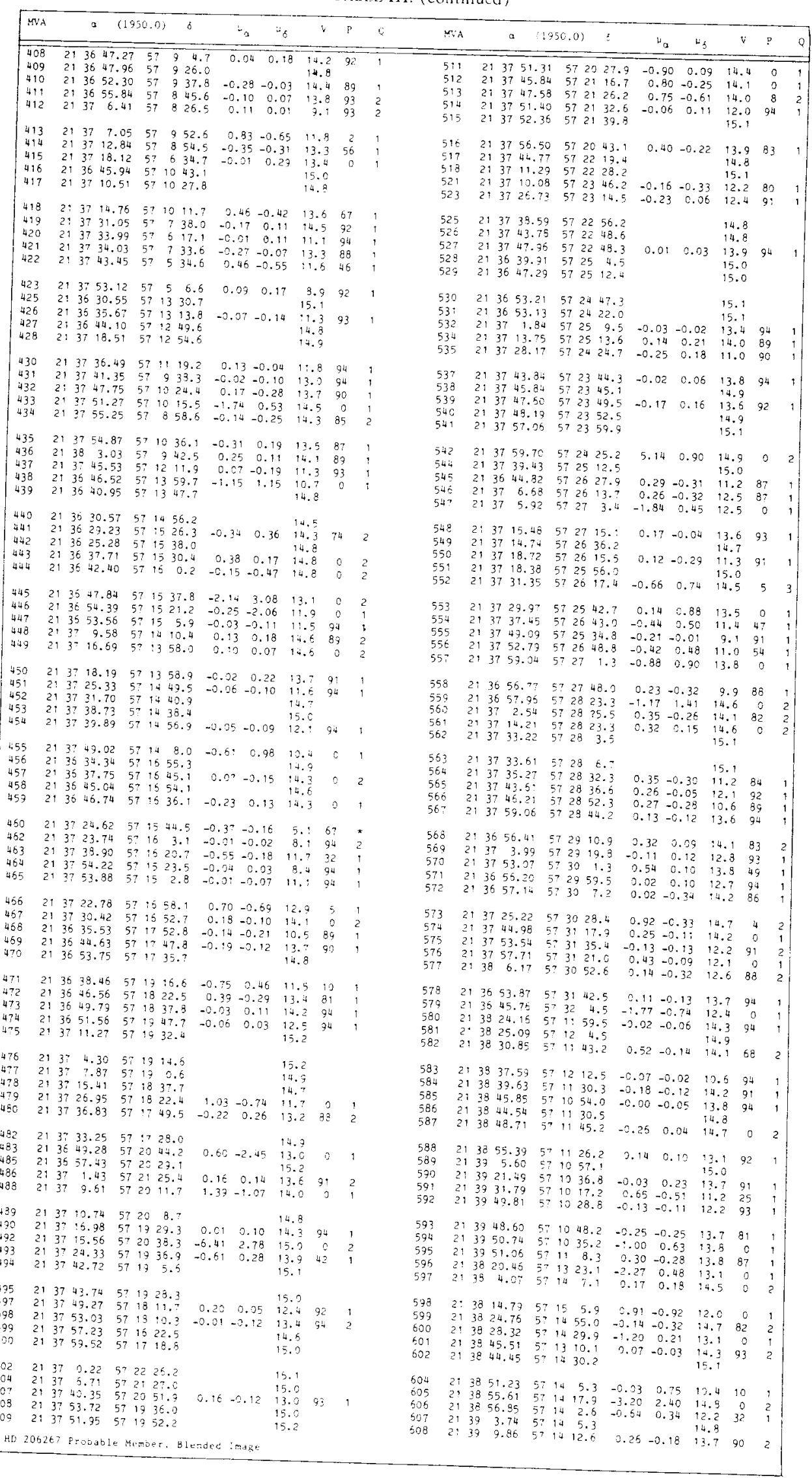


TABLE. III. (continued)

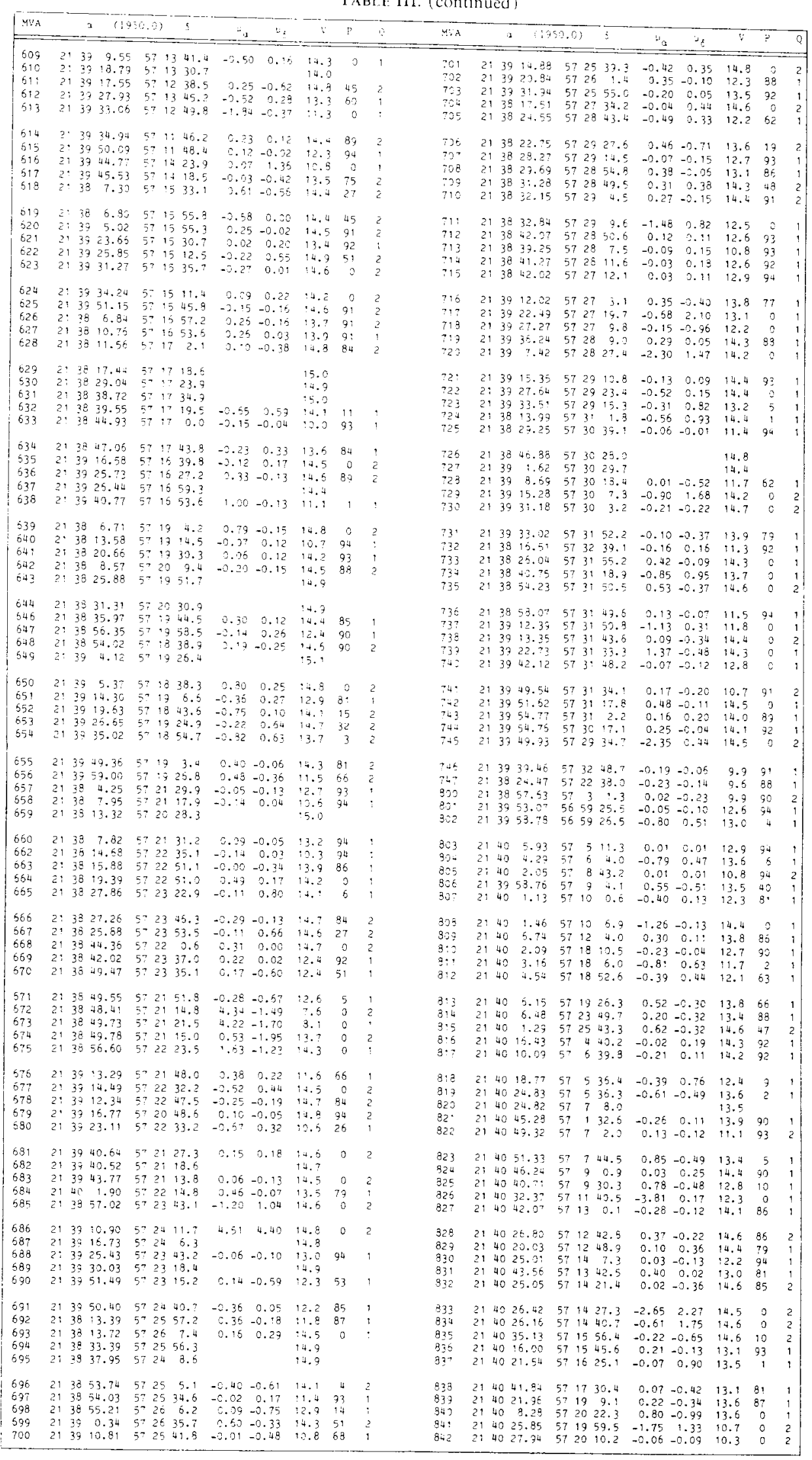


TAB1) II]. (comblnued)

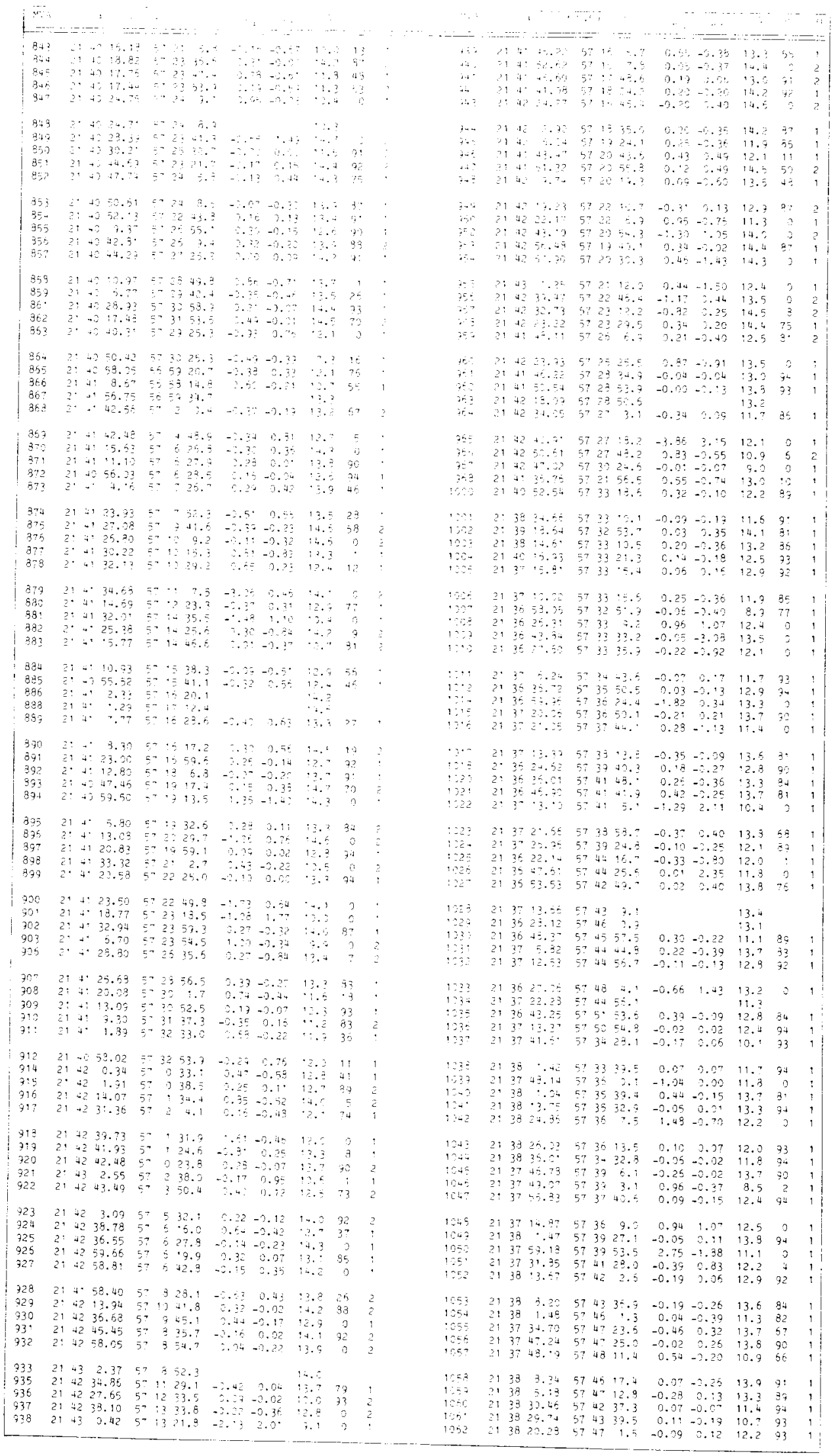


TABII: III. (continued)

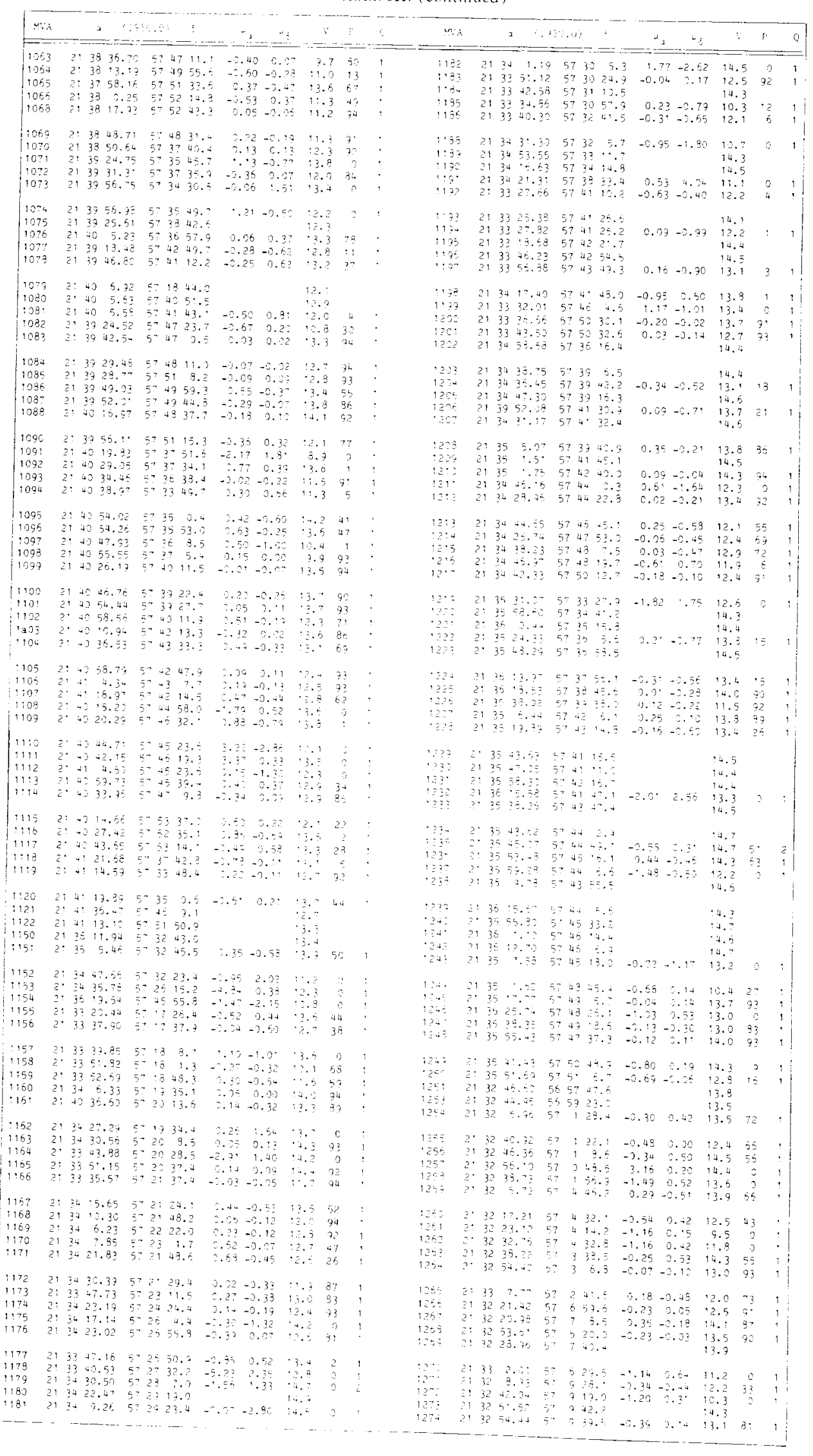


TABLF. III. (continued)

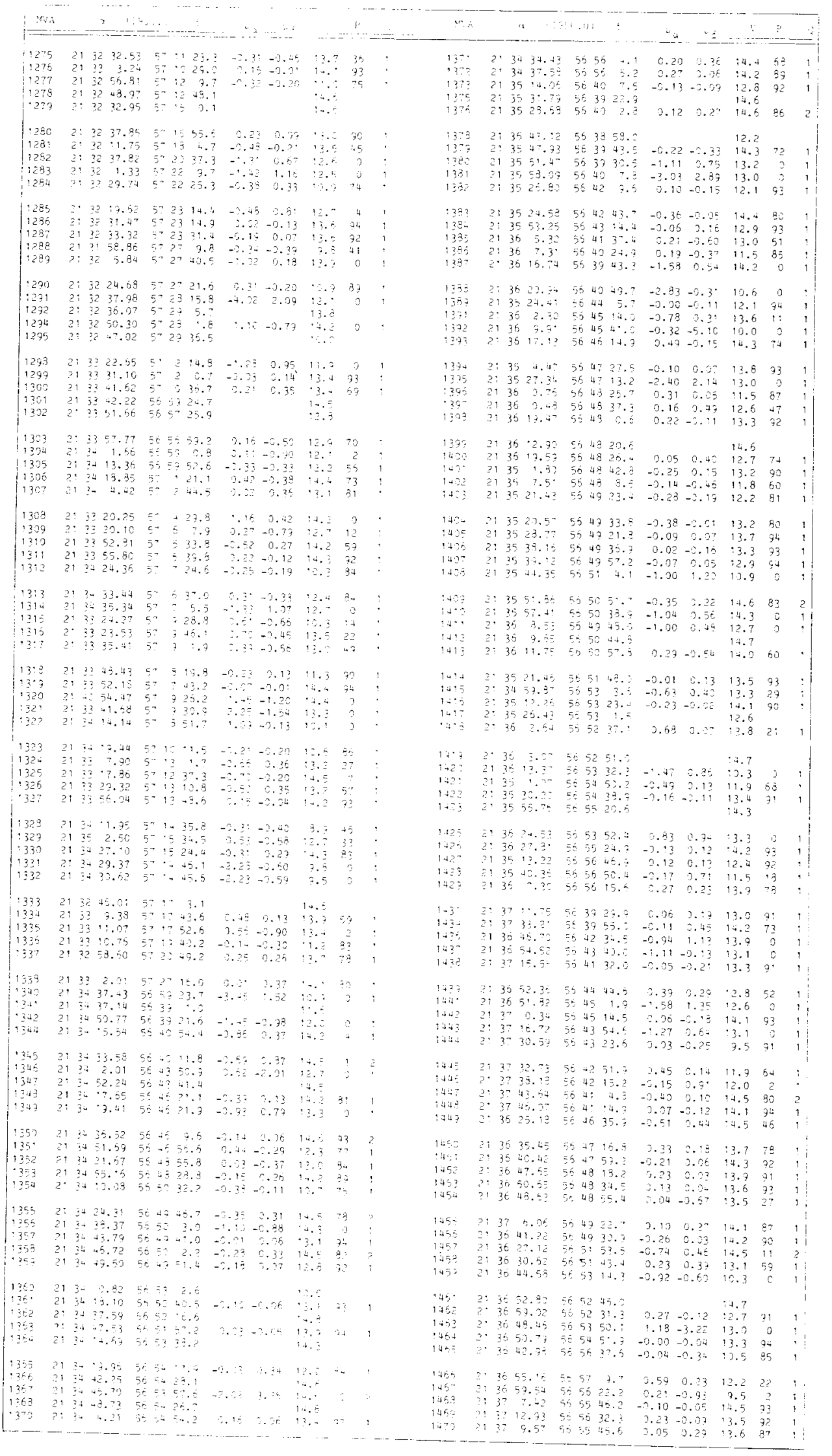


TaBI.E III. (continued)

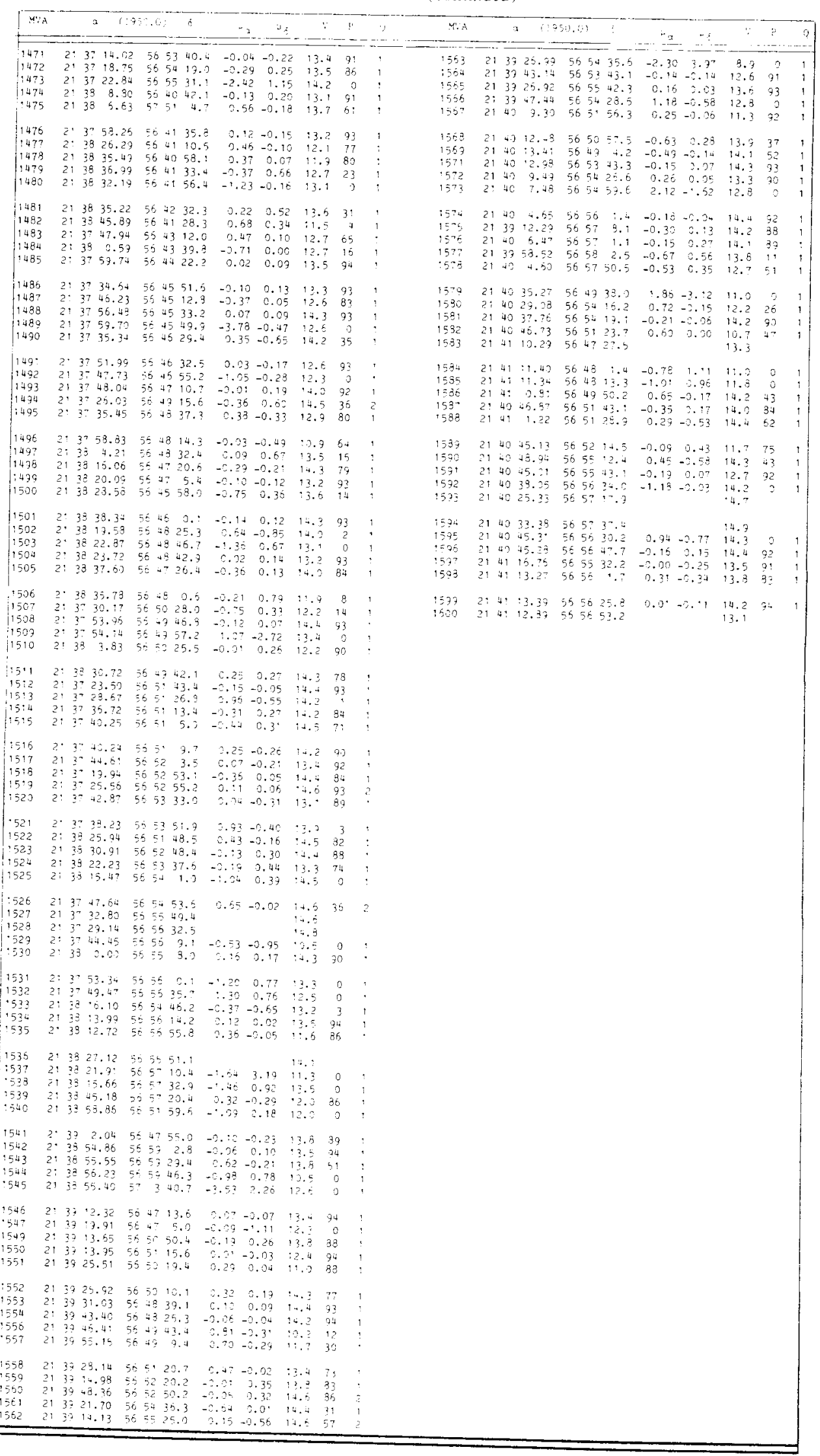




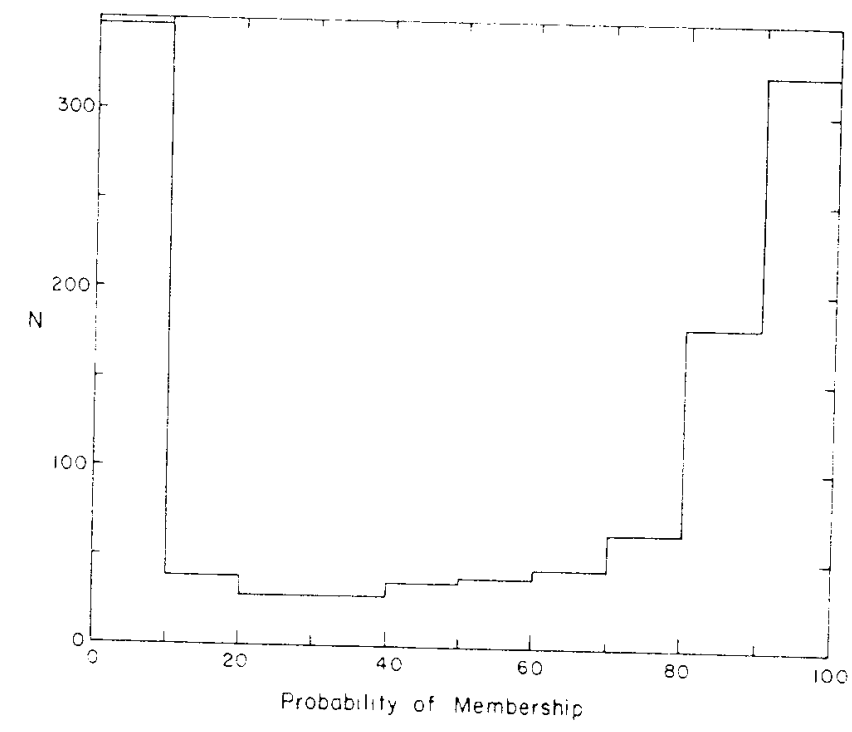

Fig. 5. Histogram of the cluster membership probahilities for all stars in the ficid with measured proper motions.

spectroscopic binary. Until further observations are obtained of this and other stars in the region. we cannot determine whether the center-of-mass velocity of MVA 550 is consistent with cluster membership. (Heske and Wendker (1986) have measured radial velocities of $\mathrm{H}_{2} \mathrm{CO}$ in the $\mathrm{IC}$ 1396 region, obtaining heliocentric velocities near Tr 37 of between -13 and $-20 \mathrm{~km} / \mathrm{s}$. The radial velocities we measure for MVA 550 are -25 , -16 , and $-6 \mathrm{~km} / \mathrm{s}$, with precision better than $\pm 0.5 \mathrm{~km} / \mathrm{s}$ for each measurement.)

Comparing our memberships with Simonson's (1968) study of Cepheus OB2, we find eight stars in common. Simonson's membership determinations depend on photometric determinations of the distance moduli of individual stars. All four of his definite members are kinematically probable members. His two "possible" members (HD $206773=$ MVA 864 and HDE $239710=$ MVA 59) are kinematic nonmembers. The remaining two stars (HDE $239724=$ MVA 412 and HDE $239727=$ MVA 423) were considered by Simonson to be distant nonmembers. We assign them high probabilities of membership. HDE 239724, a B1 V star according to Garrison and Kormendy (1976), is considered a member in their study. HDE 239727, an A2 Ia according to Garrison and Kormendy, is designated a nonmember, in agreement with Simonson but in disagreement with our results.

Garrison and Kormendy's study includes a total of 16 stars in common with ours. They determined UBV magnitudes and spectral types for all of these stars, and the derived color excesses were used to construct an H-R diagram for the cluster. Only two stars are considered nonmembers of $\operatorname{Tr} 37$ according to them: MVA 423, mentioned above; and MVA 455, a B5 Ib-II, which we determine to be a probable member. Of the remaining 14 Garrison and Kormendy stars, seven are probable kinematic members and seven are probable nonmembers. All but one (MVA 59) of the latter group (MVA 967,864,1158.1331,1328, and 1584) are found in the outer fields of our study. Two of these (HD $207049=$ MVA 967 and $H D 205850=$ MVA 1331) are giants of class B8 and $F 3$, respectively. The distance of all these stars from the cluster center lends credibility to their indicated nonmembership, but again these stars merit further scrutiny.
Most of the Ha stars selected by Kun (1986) are too faint to appear on our first-epoch plates or lie outside the region we have studied. Nevertheless, since stars of this type are generally believed to be very young objects, their membership in the cluster should afford another check of our probabilities. Of 11 common stars, seven $(63 \%)$ have kinematic membership probabilities greater than $80 \%$, one (Kun 60 $=$ MVA 1355) has a probability of $78 \%$, and the remaining three stars appear to be kinematic nonmembers. The high proportion of members among these faint red stars contrasts notably with the low proportion of members among the bright red stars identified by Alksinis

The internal velocity dispersion of an open cluster would be expected to be of the order of $1 \mathrm{~km} / \mathrm{s}$ (Mathieu 1985). For the youngest clusters, which may not be relaxed or even bound systems, somewhat larger dispersions may be found. A dispersion of $2.2 \mathrm{~km} / \mathrm{s}$ has been measured for the young cluster of stars near the Trapezium by McNamara (1976). The observed proper-motion dispersion in $X$ for $\operatorname{Tr} 37$ cluster members corresponds to a velocity dispersion at $1 \mathrm{kpc}$ of $9.5 \mathrm{~km} / \mathrm{s}$. Since this is consistent with the measurement er-

TABI I IV. Cross identifications of Alksinis and MVA stars

\begin{tabular}{|c|c|c|c|c|c|}
\hline$\therefore I K S$ & Writ & ALKS & Nit & ALKS & INA \\
\hline 158 & 1360 & 450 & 182 & 502 & 883 \\
\hline 162 & 156 & $45 ?$ & 144 & 503 & 901 \\
\hline 166 & 1420 & 452 & 194 & 505 & 898 \\
\hline 158 & 1465 & 453 & 147 & 506 & 881 \\
\hline 169 & 1459 & 454 & 153 & 507 & $9+5$ \\
\hline 171 & $\therefore-10$ & 455 & 213 & 508 & 900 \\
\hline $1: 2$ & 1467 & 456 & 468 & 509 & 936 \\
\hline$\therefore 7 .$. & $1-4+4$ & $45 ?$ & 426 & 510 & 964 \\
\hline 176 & 1529 & 458 & 471 & 511 & 938 \\
\hline 279 & 1535 & 459 & 438 & 512 & 920 \\
\hline 181 & 1537 & 460 & $54 \overline{3}$ & 703 & 1183 \\
\hline 182 & 1539 & 461 & 268 & 704 & 1191 \\
\hline i85 & $i 5++$ & $45=$ & 558 & .05 & 188 \\
\hline isd & 1503 & 463 & 412 & .08 & 1216 \\
\hline 195 & i5s9 & 464 & 273 & 709 & 1244 \\
\hline$\therefore 1$ & 2582 & 465 & 550 & $7: 1$ & 1226 \\
\hline 204 & 1594 & 460 & 460 & 712 & 252 \\
\hline 43 & 1288 & 467 & 535 & 713 & 1154 \\
\hline $4+4$ & $\therefore 21$ & 468 & 420 & 715 & 1030 \\
\hline $4+5$ & 128. & 469 & 564 & $7: 6$ & 1007 \\
\hline$\therefore 15$ & 1262 & 470 & 463 & 717 & 1022 \\
\hline+17 & 1272 & $47:$ & 437 & 718 & 1034 \\
\hline+18 & 1293 & 472 & 566 & 719 & 1016 \\
\hline 419 & 127 & 473 & 455 & 721 & $103 \%$ \\
\hline 420 & $: 270$ & 474 & 555 & 722 & 1046 \\
\hline 422 & 1336 & 475 & 423 & 223 & 1057 \\
\hline-23 & $13: 5$ & 476 & 556 & 724 & $5: 6$ \\
\hline 424 & 1166 & $47 ?$ & 454 & 726 & 1050 \\
\hline 425 & 1318 & 478 & 319 & 727 & 732 \\
\hline+26 & 1158 & 479 & 577 & 730 & 725 \\
\hline 427 & 1328 & 480 & 630 & $\div 3 !$ & 1061 \\
\hline 428 & 1322 & 481 & 662 & 732 & 1060 \\
\hline 429 & 1323 & 482 & 747 & 733 & 1001 \\
\hline-31 & $133 !$ & 483 & 318 & 734 & 1044 \\
\hline 432 & 1332 & 484 & 583 & 735 & 1063 \\
\hline 435 & 81 & 485 & 713 & 736 & 1069 \\
\hline 436 & 67 & 486 & 633 & 73 & 736 \\
\hline 437 & 68 & 488 & 800 & $7 \div 0$ & 728 \\
\hline 438 & 86 & 489 & 700 & $7 \div 1$ & 737 \\
\hline 439 & 45 & 490 & 680 & 742 & 1082 \\
\hline$\angle 40$ & 63 & 491 & 638 & 743 & 745 \\
\hline 441 & 59 & 492 & $6 ! 6$ & 744 & $\because 41$ \\
\hline 442 & 71 & 493 & 656 & 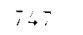 & $109 i$ \\
\hline $4+3$ & 90 & 495 & 811 & 748 & 1110 \\
\hline $4-4$ & $10 !$ & 496 & 812 & 749 & 1097 \\
\hline$+4 j$ & 26 & 497 & 846 & 750 & 864 \\
\hline 445 & 186 & 498 & 847 & 751 & 1098 \\
\hline 447 & 106 & 499 & 850 & 752 & 908 \\
\hline $4+8$ & 181 & 500 & 822 & 703 & 967 \\
\hline 449 & 164 & 501 & 903 & & \\
\hline
\end{tabular}


rors in our previous study of NGC 2244, scaled roughly by the square root of the number of plates in the solutions, we conclude that this dispersion is almost entirely accounted for by random error and does not significantly reflect the internal kinematics of the cluster. More plate material over a longer baseline, or alternatively radial-velocity studies such as those reported by Mathieu (1985), will be required for such investigations.

The only anomaly in the observed proper-motion distribution is the inequality between the dispersion of the proper motions in $X(0.19$ arcsec/century $)$ and in $Y(0.28$ arcsec /century). We have investigated a number of sources of error in our measurement and reduction procedure and have so far been unable to determine the cause of the discrepancy. It should be noted that the $X$ and $Y$ dispersions refer to frame rotated $48^{\circ}$ to right ascension and declination (see Fig. 2) so as to align the major and minor axes of the field distribution with the coordinate axes. Thus the observed discrepancy cannot be accounted for by an elongation of the star images in right ascension due to improper guiding. or a failure to account for refraction effects in the plate solution. The larger $Y$ dispersion is in the direction of the galactic plane, and it is possible that the distribution of field proper motions in this direction has contaminated the cluster distribution. Nevertheless, the discrepancy between $X$ and $Y$ dispersions was also noted on plate solutions utilizing only brighter stars in the inner regions of the center plates, where one would expect field-star contamination to be diminished. Better proper motions, using new plates at a later epoch, might resolve the issue. The alternative possibility, that the cluster is expanding along the galactic plane, seems untenable, since the velocity of expansion implied by the $Y$ dispersion, almost $14 \mathrm{~km} / \mathrm{s}$, would be unrealistically high, as we noted earlier.

Whatever the cause of the discrepancy in the $X$ and $Y$ dispersions, its effect is that our proper-motion membership probabilities are based more on the $X$ proper motions than the $Y$, but the agreement with other criteria of cluster membership that we have discussed previously bears out the adequacy of the kinematic probabilities. Until a source of the discrepancy is identified, however, interpretation beyond the use of membership probabilities should be made with caution.

No comprehensive photometric or spectroscopic studies exist for the entire region at the scale at which we have studied it, but we can infer from the similarity between the appearance of $\operatorname{Tr} 37$ and NGC 2244 that the former cluster is as entangled with absorbing material as the latter, and that dif- ferential absorption across the cluster is likely to cause significant uncertainties in interpreting the spatial distribution, the luminosity function, or the color-magnitude diagram of the cluster (Marschall et al. 1982). In the case of IC 1805. which is also similar in age and appearance to $\operatorname{Tr} 37$ and NGC 2244, Johnson et al. (1961) noted variations in $\mathrm{E}(B-V)$ ranging from 0.62 to 0.98 , and Vasilevskis et al. (1965) remarked on the scatter this introduced into the color-magnitude diagram of the cluster even when members were selected by proper-motion criteria. Moreover, a recently published study of ultraviolet extinction in the region around $\operatorname{Tr} 37$ (Clayton and Fitzpatrick 1987) indicates considerable variation in the properties of absorbing material across the cluster. We therefore attempt no such color-magnitude analysis here, pending the results of a photoelectric photometric study of the cluster members currently being undertaken by the authors.

Nevertheless, the selection of probable member stars. together with the coordinates and identification charts provided here. should encourage further studies of the population of this relatively accessible young cluster. A set of even larger-scale finding charts for the region, 15 arcmin on a side, especially useful for identifying stars on a TV guider, is available from the authors.

\section{V.SLMMARY}

Positions for 1387 stars, along with 1135 proper motions, have been presented for a field of $1.5^{\circ}$ diameter including the cluster Trumpler 37 . Stars in this sample with derived membership probabilities of $>80 \%$ should provide a reliable subset for further studies of the stellar content and internal kinematics of the cluster.

We thank Drs. Kyle Cudworth and Bob Mathieu for several helpful discussions. Dr. E. Green kindly provided the improved cluster probability programs. Rob Becker and Jim Neilson carried out much of the labor of data entry. The Gettysburg College Computer Center provided time on its B6700 computer for the membership analysis and word processing. We also thank Dr. David Latham and the Center for Astrophysics, Cambridge, MA, for hospitality to one of us (L,M.) during the writing of this paper. This research was carried out in part under NSF grants AST 8311337 and AST 8503236, and with additional support from the NSF-ROA grant program and the Gettysburg College Faculty Self-Renewal Fund.

\section{REFERENCES}

Abt. H. A. (1986). Astrophys. J. 304, 688.

Alksinis, A. (1958). Lan Trans. 7,33.

Barnard, E. E. (1927). A Photographic Atlas of Selected Regions of the

Milky Way (Camegie Institution of Washington. Washington, DC). plate 49.

Cardon de Lichtbuer, P. (1982). Vat. Obs. Publ. 2, 1

Clayton, G. F., and Fitzpatrick. E. L. (1987). Astron. J. 92. 157

Cudworth. K. M. (1986). Astron J. 91, 348.

Garrison, R. F, and Kormendy. J. (1976). Publ. Astron. Soc. Pac. 88, 865

Heske, A., and Wendker, H. J. (1986). Astron. Astrophys. 149. 199.

Johnson, H. L.. Hoag. A. A., Iriarte, B., Mitchell, R. I., and Hallam. K. L.

(1961). Lowell Obs. Bull. 5. 133.

Kun. M. (1986). Astrophys. Space Sci. 125.13
Lynga, G. (1985), Lund Catalogue of Open Clusters, th ed. (Stellar Data Center, Strasbourg).

Marschall, L., van Altena. W. F, and Chiu, L.-T. G. (1982), Astron J. 87, 1497

Mathieu, R. D. (1985), In Dynamics of Star Clusters, edited by J. Goodman and P. Hut (Reidel, Dordrechn). p. 427.

Simonson, S. C. (1968). Astrophys. J. 154, 923.

Trumpler. R. J. (1930). Lick Obs. Bull. 14, 420.

van Altena, W. F and Jones, B. F. (1972). Astron. Astrophys. 20. 425

Vasilerskis, S. Sanders, W. L., and van Altena, W. F. (1965). Astron. J. 70, 806.

Wendher, H. J.. and Batrs. J. W. M. (1980). Astron. Astrophys. 89, 180. 